\title{
SEGUIMIENTO Y EVALUACIÓN DE LAS PRÁCTICAS DE ENFERMERÍA ATRAVÉS DE LA APLICACIÓN SISEP
}

\section{MONITORING AND EVALUATION OF NURSING PRACTICES THROUGH THE SISEP APPLICATION}

\author{
Vanesa Cantón-Habas ${ }^{1}$, María del Pilar Carrera-González ${ }^{2}$, Sergio David \\ Sánchez-Ortiz ${ }^{3}$, Pilar María Lora-López ${ }^{3}$, Juan Crisóstomo Vacas-Pérez ${ }^{3}$, \\ Mercedes López-Pardo Martínez ${ }^{3}$, Inés Carmen Rodríguez-García ${ }^{3}$, Manuel \\ Toledano-Estepa ${ }^{3}$, Manuela Urbano-Priego ${ }^{3}$, Carlos José Pérula-de-Torres ${ }^{3}$, \\ Jose Hernández-Ascanio ${ }^{3}$, Manuel Rich-Ruiz ${ }^{4}$.
}

Fecha de recepción: 25/11/2020; Fecha de revisión: 16/01/2020; Fecha de aceptación: 19/02/2020

Cómo citar este artículo:

Cantón-Habas, V., Carrera-González, M.D, Sánchez-Ortiz, S.D., Lora-López, P.M, Vacas-Pérez, J.M., López-Pardo Martínez, M., Rodríguez-García, I.C., Toledano-Espeta, M., Urbano-Priego, M., Pérula-deTorres, C.J., Hernández-Ascanio, J., Rich-Ruiz, M. (2020). Seguimiento y evaluación de las prácticas de enfermería a través de la aplicación SISEP. Revista de Innovación y Buenas Prácticas Docentes, 9(1), 5360.

\section{Autor de Correspondencia: n92cahav@uco.es}

\section{Resumen:}

El avance tecnológico y la consecuente incorporación de las TICs en el ámbito docente ha conllevado la aparición de nuevas herramientas para facilitar a los docentes el proceso de seguimiento y evaluación del alumnado. De igual forma, dicho avance ha contribuido a la adquisición de un modelo de aprendizaje más proactivo e individualizado por parte del alumnado. El presente proyecto pretende implementar una herramienta informática denominada "Seguimiento y Evaluación de Practicas" (SISEP) en las asignaturas de prácticas clínicas de la titulación de Grado en Enfermería para optimizar el seguimiento y la evaluación de los alumnos durante el desarrollo de sus prácticas externas, así como mejorar el proceso de adquisición de competencias y habilidades. La metodología ha consistido en la elaboración de material didáctico para dicha aplicación y la posterior evaluación del grado de satisfacción de los distintos roles implicados en su uso mediante un cuestionario autoadministrado de elaboración propia. El grado de satisfacción con respecto a la aplicación fue alto, señalando que contribuye de forma decisiva en la adquisición de competencias, aunque no en todos los casos los usuarios han considerado su interfaz como completamente sencilla o intuitiva.

Palabras clave: desarrollo competencial, enfermería, prácticas clínicas, TICs.

\footnotetext{
${ }^{1}$ Universidad de Córdoba (España), n92cahav@uco.es; CÓDIGO ORCID: https://orcid.org/0000-00023928-0092

2 Universidad de Córdoba (España), pcarrera@uco.es

${ }^{3}$ Universidad de Córdoba (España)

${ }^{4}$ Universidad de Córdoba (España), en1rirum@uco.es
} 


\section{Abstract:}

The technological advance and consequent incorporation of IT in the teaching field has entailed the appearance of new tools to facilitate teachers the process of monitoring and evaluating students. In the same way, this advance has contributed to the acquisition of a more proactive and individualized learning model on the students' side. The present project intends to implement a computer tool called "Monitoring and Evaluation of Practices" (SISEP) in the subjects of clinical practices of the degree in Nursing in order to optimize the monitoring and evaluation of students during the development of their external practices, as well as improving the process of acquiring skills and abilities. The methodology used consisted in the elaboration of the teaching material and the later evaluation of the degree of satisfaction from the different roles involved through the use of a self-administered, self-produed questionnaire. The degree of satisfaction regarding the application was high, pointing out its decisive contribution to the acquisition of skills, even if not all users have found its interface clear or intuitive enough.

Key Words: Competential development, clinical practices, nursing, ICT 


\section{INTRODUCCIÓN}

Enfermería es una profesión eminentemente práctica (Heydari, Kamran, Mohammady, \& Hosseinabadi 2012; Huybrecht, Loeckx, Quaeyhaegens, De Tobel, \& Mistiaen, 2011). A pesar de esta concepción desde la perspectiva universitaria e institucional ha venido existiendo un notable interés por disminuir la separación entre el conocimiento teórico y el práctico, en el que durante muchos años hemos estado enmarcados (Amezcua \& González-Iglesias, 2015). La creación del Espacio Europeo de Educación Superior (EEES) supuso una profunda transformación de la educación universitaria, permitiendo a los profesionales de enfermería lograr una importante promoción de la disciplina. Asimismo, esta nueva conceptualización de la profesión enfermera permitió desde el ámbito docente consolidar la disminución de dicha separación de conocimientos (Martínez, 2007).

Dentro de la titulación de enfermería, los Practicums son asignaturas de carácter obligatorio cuyo objetivo es otorgar al alumno de un aprendizaje profesional, configurados estos desde una perspectiva de complejidad creciente y teniendo en cuenta las diversas áreas de competencia profesional (Rodicio \& Iglesias, 2011). Por consiguiente, en el contexto del EEES, el Practicum abandona el papel secundario otorgado en plantes formativos anteriores, adquiriendo una mayor relevancia y pasando de ser periodos formativos prácticos englobados dentro de las asignaturas teóricas a ser un componente más de la titulación con un importante número de créditos asociado (Martínez \& Raposo, 2011).

Las prácticas clínicas contribuyen a que los alumnos desplieguen el conocimiento científico teórico en el contexto asistencial mediante la integración del conocimiento teórico y teórico-práctico adquirido en las aulas, así como en las salas de simulación, permitiendo estas el desarrollo de actitudes profesionales (Price, Hastie, Duffy, Ness, \& McCallum, 2011). De esta forma, el conocimiento teórico permite asentar las bases y directrices, mientras que la práctica habilita el desarrollo de habilidades y aptitudes en el ambiente profesional real (Zabalza, 2011).

La importancia de la formación práctica reside en que es la estrategia que permite al alumno ejercitar diversas destrezas y conocimientos, siendo estos elementos fundamentales para aprender el desarrollo de su futura profesión.

Desde la implantación de la Titulación de Grado en Enfermería englobada dentro del EEES, el seguimiento del desarrollo de estos periodos formativos, así como su evaluación ha estado basado en la adquisición de una serie de competencias básicas y específicas, las cuales dependerán del ámbito de desarrollo de las mismas, así como de la complejidad de la atención al paciente-, utilizando como instrumentos principales para la evaluación el control de asistencia a prácticas, escalas de aptitudes y diario reflexivo y/o portfolio, todas ellas en formato papel (González-Hernando, Martín-Villamor, Carbonero-Martín, \& Lara-Ortega, 2014).

Gracias al importante avance tecnológico y la incorporación de las TICs en el ámbito docente han surgido nuevas herramientas que pueden facilitar a los docentes el proceso de seguimiento y evaluación del alumnado, así como a estos permitirles seguir un modelo de aprendizaje más proactivo e individualizado (Salinas, 2004). Un ejemplo de esto es la aplicación SISEP (Seguimiento y Evaluación de Prácticas).

La aplicación informática SISEP es un instrumento idóneo para poder realizar un seguimiento exhaustivo del desarrollo de las competencias que cada alumno debe alcanzar durante su periodo formativo en prácticas según la Orden CIN/2134/2008 del 3 de julio.

En esta aplicación web cada uno de los perfiles -responsable del Practicum, 
coordinador, tutor clínico y alumno- tienen funciones específicas propias del desempeño de su rol.

Por lo tanto, esta aplicación permite que se valore de forma continuada la adquisición de habilidades por parte de este, al mismo tiempo que posibilita al profesor o tutor responsable de la evaluación para poder realizar un seguimiento pormenorizado del desarrollo de los diversos elementos a tener en cuenta en la evaluación. Asimismo, facilita un proceso de retroalimentación con el alumno permitiéndole a este conocer los elementos con posibilidad de mejora.

\section{DESARROLLO DE LA EXPERIENCIA DE INNOVACIÓN}

El presente proyecto pretende apoyar la implantación de una herramienta informática para el seguimiento de la adquisición de competencias dentro de las asignaturas Practicum I, II, III, IV, V, VI, VII y VIII de la titulación de Grado en Enfermería. Para ello se ha realizado:

- Confección de los documentos necesarios para la implementación de la herramienta web para el seguimiento de las prácticas del Grado en Enfermería.

- Implementación de la aplicación informática online dentro del entorno de las asignaturas Practicum I, II, III, IV, V, VI, VII y VIII en la titulación Grado en Enfermería.

- Evaluación de la utilidad, la viabilidad y el grado de satisfacción de la aplicación informática.

Durante el curso académico 2016-2017 se procedió a la confección del material necesario para la configuración de las asignaturas y se planificó e implantó la aplicación informática online basada en la interactividad tecnológica y en la aplicación de conocimientos teóricos a la práctica en el contexto de las asignaturas anteriormente mencionadas.

- En relación con el primero de los objetivos propuestos, se han realizado reuniones de forma periódica entre los responsables de los Prácticums para elaborar documentos homogenizados tanto en lo referido a la consecución de competencias y habilidades por parte del alumnado como a la creación de rúbricas de evaluación establecidas mediante consenso. Además de las reuniones presenciales, ha sido necesario un proceso de comunicación constante entre el responsable del proyecto y los demás participantes.

- Derivado del segundo de los objetivos, se inició la implementación de la aplicación informática en las asignaturas de prácticas del primer cuatrimestre del citado curso académico, concretamente esto aconteció en los Prácticums V, VI y VII, de los cuales dos son realizados en el contexto de atención primaria y el último de estos en atención hospitalaria maternal y pediátrica. En el segundo cuatrimestre se procedió a la implementación de esta en los Prácticum I, II, III, IV y VIII, correspondiendo estos periodos de prácticas mayoritariamente a atención hospitalaria.

- Finalmente, con respecto al tercer objetivo, para evaluar la utilidad, la viabilidad y el grado de satisfacción con la aplicación informática se solicitó la cumplimentación de una encuesta con carácter anónimo y voluntario, de elaboración propia y consensuada con un grupo de expertos, a todos los usuarios que habían utilizado dicha herramienta, habiendo obtenido de esta forma información del profesorado responsable de los prácticums $(n=6)$, de los profesores coordinadores $(n=6)$, de los tutores clínicos $(n=5)$ y de los propios alumnos $(n=30)$. 


\section{RESULTADOS}

Fruto de las reuniones entre los distintos responsables se diseñaron los elementos claves para el funcionamiento de la aplicación informática:

- Base de datos de hospitales, centros y servicios

- Base de datos de profesorado y tutores clínicos implicados en el aprendizaje del alumnado

- Base de datos con información referente a las intervenciones y actividades de enfermería, basadas en la Taxonomía NIC (Bulechek, \& Butcher, 2013), que los alumnos deben desarrollar durante cada una de las asignaturas.

- Rúbricas de evaluación

En base a la información que los distintos sujetos que han colaborado en la utilización de esta herramienta facilitaron a través de la cumplimentación de la encuesta confeccionada para valorar la utilidad, la viabilidad y el grado de satisfacción con ella, se obtuvieron los resultados que se desarrollan a continuación.

Respecto al diseño y la facilidad de uso el $63 \%$ del alumnado expuso que el manejo de la herramienta informática web era intuitivo frente al 37\% que manifestó que en ocasiones no lo era. De igual forma, el 30\% del alumnado manifestó estar totalmente de acuerdo con la afirmación "Ios contenidos de la aplicación y materiales del programa se comprenden sin dificultad" frente al $43 \%$ que expresaron no estar de acuerdo con la misma, y sólo el $27 \%$ manifestó no estar ni en acuerdo ni en desacuerdo con dicha afirmación.

Respecto a la metodología de aprendizaje, el 43\% del alumnado consideraba que la aplicación le parecía muy útil para la consecución de las competencias de las prácticas externas, el 28\% manifestó estar de acuerdo con que la aplicación era útil para dicha consecución, frente al $29 \%$ que manifestó no estar ni de acuerdo ni en desacuerdo. Asimismo, el $82 \%$ de los alumnos consideraron que la aplicación informática incluye un lenguaje enfermero entendible y útil para alcanzar las destrezas necesarias durante dicha asignatura.

El 86\% del alumnado consideró que la utilización de la aplicación permite un seguimiento más continuado y el $87 \%$ expresó que la utilización de la aplicación permite un seguimiento individualizado. Además, el 100\% de los alumnos afirmó que la aplicación ha permitido objetivar la evaluación obtenida.

En términos generales, el grado de satisfacción con la aplicación fue elevado, ya que un $24 \%$ la consideraba muy buena. Un $72 \%$ que expresó que recomendaría la utilización de esta al resto de sus compañeros.

El ítem menos favorable es que, respecto a la afirmación "el diseño de la interfaz de usuario se caracteriza por claridad y sencillez permitiendo un uso adecuado de la aplicación informática", el 71\% del alumnado que ha utilizado la aplicación mostró no estar ni en acuerdo ni en desacuerdo, frente a un 29\% que manifestó estar de acuerdo con esta afirmación, por tanto, ningún alumno manifestó que la aplicación tenía una interfaz especialmente completa para su utilización.

Respecto a la opinión de los tutores clínicos que de forma voluntaria han querido participar en la implementación de esta herramienta en el contexto de las prácticas clínicas de los alumnos de Grado en Enfermería, cabe destacar que el 100\% afirmó que la aplicación informática era sencilla e intuitiva, y de gran utilidad para el 
proceso de aprendizaje del alumnado.

En términos generales, el $100 \%$ de los tutores clínicos recomendarían su utilización al resto de sus compañeros; sin embargo, estos evidenciaron que, en ocasiones, a pesar de que el tiempo que deben emplear en la misma es escaso, la utilización de ésta dentro de su labor profesional se ve dificultada por la carga asistencial existente, motivo por el que consideran que la aceptación de esta herramienta dentro del colectivo enfermero se verá modificada de forma negativa.

De igual forma, el $100 \%$ de los profesores participantes en la implantación de la aplicación informática consideraban de utilidad la utilización de esta para la consecución de las competencias de la asignatura, así como que esta incluye un lenguaje enfermero entendible y útil para alcanzar las destrezas necesarias durante la formación en dichas asignaturas.

El 100\% también consideró que la aplicación web permite un seguimiento más continuado e individualizado del alumno, y que la implementación de esta herramienta informática permite objetivar la calificación obtenida por los alumnos.

En términos generales, el 100\% del profesorado recomendaría la utilización de la aplicación informática al resto de sus compañeros.

En relación a la opinión de los responsables de las asignaturas, el 100\% de estos consideraba que el interfaz de usuario se caracteriza por ser sencillo permitiendo un uso adecuado de la aplicación informática. Sin embargo, solo el 50\% consideraba que su manejo era intuitivo.

De igual forma, el 100\% de los responsables de las asignaturas consideró que la aplicación le parece útil para la consecución de las competencias de la asignatura, así como que esta incluye un lenguaje enfermero entendible y útil para alcanzar las destrezas necesarias durante dicha asignatura.

Respecto al trabajo en equipo, el $80 \%$ de estos expusieron que consideraban que la aplicación favorece el trabajo en equipo entre tutores clínicos, profesores y alumnado para conseguir una mejora en el aprendizaje del alumno, frente a un $20 \%$ que no estaba de acuerdo ni en desacuerdo con esto. No obstante, en las preguntas de respuesta abierta un elevado porcentaje de profesores manifestaron que en la actualidad el mayor impedimento para que la aplicación se pueda continuar utilizando de forma que repercuta positivamente en el aprendizaje del alumno se debe a la reducida implicación de los diversos roles en dicho proceso de aprendizaje.

Un 50\% del profesorado manifestó estar de acuerdo con la afirmación "el diseño de la interfaz de usuario se caracteriza por claridad y sencillez permitiendo un uso adecuado de la aplicación informática" frente al otro 50\% quienes expusieron no estar de acuerdo ni en desacuerdo con dicha afirmación, y el 100\% se mostraron ni en acuerdo ni en desacuerdo con que el manejo de la misma era intuitivo. Por otro lado, todos expusieron que los contenidos y materiales del programa se comprendían sin dificultad.

El 50\% manifestó estar totalmente de acuerdo con la afirmación "la utilización de la aplicación permite un seguimiento más continuado del alumnado" frente al 50\% que expuso estar de acuerdo con la misma. De igual forma, el 100\% de los profesores responsables de los prácticums manifestaron que la aplicación permite un seguimiento individualizado del alumno y que esto ha repercutido positivamente en la objetivación de las calificaciones.

Finalmente, en relación con la información facilitada por los responsables de las asignaturas, cabe destacar que todos recomendarían la utilización de la aplicación informática y su grado de satisfacción con esta fue alto.

En base a lo anteriormente expuesto y en concordancia con lo evidenciado por diversos autores, en consonancia con la información revelada por los participantes en el presente proyecto de innovación docente, la implementación de herramientas informáticas dentro de la docencia favorece la adquisición de las competencias por parte del alumnado (García-Marcos, \& Cabero-Almenara, 2016). La incorporación paulatina de estas al ámbito docente conlleva efectos positivos a todos los usuarios 
involucrados en su utilización, a pesar de la posible reticencia existente en un inicio de su implementación por la desconfianza que genera el cambio de la metodología tradicionalmente empleada.

La reticencia expresada por los tutores clínicos con respecto a la viabilidad de la implementación final del uso de la aplicación fuera del ámbito de este proyecto es debida a la sobrecarga asistencial que aseguran sufrir actualmente, al pensar que el tener que gestionar la aplicación no hará sino incrementar su carga laboral (Yu, Raphael, Mackay, Smith, \& King, 2019). Por lo tanto, la extensión final del uso de la aplicación al total del alumnado de Grado de Enfermería estará limitada por la implicación de los tutores clínicos en el proceso de aprendizaje, y de forma indirecta interferirá con el grado de satisfacción y la adquisición de competencias por parte de los alumnos. Sin embargo, cabe destacar que los cuatro grupos que forman parte de este proyecto -entre los que se engloban los tutores clínicos a los que se hace referencia- han evidenciado la utilidad de la aplicación a la hora de objetivar el proceso de desarrollo de habilidades y evaluación de las mismas.

Como principales limitaciones destacan la complejidad del desarrollo del material didáctico y las rúbricas de evaluación consensuadas para todos los periodos de prácticas externas -indistintamente de que se traten de ámbito hospitalario o de atención primaria-, además de su extensión para su uso por parte de todo el alumnado, tutores clínicos y profesorado. Por el contrario, como fortalezas, es de necesidad destacar el auge de las TICs sobre métodos tradicionales como la escritura en papel, repercutiendo de forma positiva en el seguimiento realizado al alumnado, así como en la objetivación de las calificaciones.

\section{CONCLUSIONES}

La implementación de una herramienta informática para el seguimiento y evaluación del alumnado en el contexto de las prácticas clínicas de enfermería ha permitido crear y adaptar el material didáctico a las nuevas tecnologías. Mediante la evaluación de esta experiencia de innovación se ha evidenciado que la utilización de la aplicación informática es una estrategia que ha contribuido significativamente a mejorar el proceso de aprendizaje, basada en la adquisición de competencias reflejadas mediante la utilización del lenguaje enfermero y en un seguimiento exhaustivo e individualizado del alumnado, habiendo contribuido de forma decisiva en la objetivación del proceso de evaluación.

\section{REFERENCIAS}

Amezcua, M., \& González-Iglesias, M.E. (2015). La creación del título de Enfermera en España: ¿cien años de una incoherencia histórica?. Index de Enfermería, 24(12), 79.

Bulechek, G.M., \& Butcher, H.K. (2013). Clasificación de Intervenciones de Enfermería (NIC) (6 Edición). Madrid: Elsevier.

García-Marcos, C.J, \& Cabero-Almenara, J. (2016). Evolución y estado actual del elearning en la Formación Profesional española. Revista Iberoamericana de Educación a Distancia, 19.

González-Hernando, C., Martín-Villamor, P., Carbonero-Martín, M.A., \& Lara-Ortega, C. (2014). Nursing student's competency assessment through Problem Based Learning. Enfermería Universitaria, 120-124. 
Heydari, H., Kamran, A., Mohammady, R., \& Hosseinabadi, R. (2012). The experiences of nursing students of the mentorship program: a qualitative study. Health System Research, 8(3), 438-48.

Huybrecht, S., Loeckx, W., Quaeyhaegens, Y., De Tobel, D., \& Mistiaen, W. (2011). Mentoring in nursing education: perceived characteristics of mentors and the consequences of mentorship. Nurse Education Today, 31(3), 274-8. doi: 10.1016/j.nedt.2010.10.022.

Martínez, E. \& Raposo, M. (2011). Funciones generales de la tutoría en el Practicum: entre la realidad y el deseo en el desempeño de la acción tutorial. Revista de Educación, 354,155-181.

Martínez, M.L. (2007). 30 años de evolución de la formación enfermera en España. Educación Médica, 10(2), 93-6.

Price, L., Hastie, L., Duffy, K., Ness, V., \& McCallum, J. (2011). Supporting students in clinical practice: Preregistration nursing students' views on the role of the lecturer. Nurse Education Today,31(8), 780-784. doi: 10.1016/j.nedt.2011.04.009

Rodicio, M.L. \& Iglesias, M. (2011). La formación en competencias a través del Practicum: un estudio piloto. Revista de Educación, 354, 99-124.

Salinas, J. (2004). Innovación docente y uso de las TIC en la enseñanza universitaria. Revista de Universidad y Sociedad del Conocimiento (RUSC), 1(1). doi: 10.7238/rusc.v1i1.228

Yu, F., Raphael, D., Mackay, L., Smith, M., \& King, A. (2019). Personal and workrelated factors associated with nurse resilience: a systematic review. International Journal of Nursing Studies, 93, 129-140. doi: 10.1016/j.jinurstu.2019.02.014

Zabalza, M. (2011). El practicum en la formación universitaria: estado de la cuestión. Revista de Educación, 354, 21- 43. 